\title{
Weighted Average Based Differential Quadrature Method for One-Dimensional Homogeneous First Order Nonlinear Parabolic Partial Differential Equation

\author{
Kedir Aliyi Koroche
}

\begin{abstract}
In this paper, the weighted average-based differential quadrature method is presented for solving one-dimensional homogeneous first-order non-linear parabolic partial differential equation. First, the given solution domain is discretized by using uniform discretization grid point. Next, by using Taylor series expansion we obtain central finite difference discretization of the partial differential equation involving with temporal variable associated with weighted average of partial derivative concerning spatial variable. From this, we obtain the system of nonlinear ordinary differential equations and it is linearized by using the quasilinearization method. Then by using the polynomial-based differential quadrature method for approximating derivative involving with spatial variable at specified grid point, we obtain the system of linear equation. Then they obtained linear system equation is solved by using the $L U$ matrix decomposition method. To validate the applicability of the proposed method, two model examples are considered and solved at each specific grid point on its solution domain. The stability and convergent analysis of the present method is worked by supported the theoretical and mathematical statements and the accuracy of the solution is obtained. The accuracy of the present method has been shown in the sense of root mean square error norm $L_{2}$ and maximum absolute error norm $L_{r \pi}$ and the local behavior of the solution is captured exactly. Numerical versus exact solutions and behavior of maximum absolute error between them have been presented in terms of graphs and the corresponding root mean square error norm $L_{2}$ and maximum absolute error norm $L_{\text {rr }}$ presented in tables. The present method approximates the exact solution very well and it is quite efficient and practically well suited for solving the non-linear parabolic equation. The numerical result presented in tables and graphs indicates that the approximate solution is in good agreement with the exact solution.
\end{abstract}

Keywords: Non-linear parabolic equation, Burger equation, Differential quadrature method, weighted average, Stability and Convergent Analysis, Root mean square and Maximum absolute error.

\section{INTRODUCTION}

Manuscript received on 04 April 2021 | Revised Manuscript received on 08 April 2021 | Manuscript Accepted on 15 April 2021 | Manuscript published on 30 April 2021.

* Correspondence Author

Kedir Aliyi Koroche*, Department of Mathematics, College of Natural and Computational Sciences, Ambo University, Ambo, Ethiopia. Email: kediraliyi39@gmail.com

(c) The Authors. Published by Lattice Science Publication (LSP). This is an open access article under the CC-BY-NC-ND license (http://creativecommons.org/licenses/by-nc-nd/4.0/)
The quasilinear parabolic partial differential equation is the simplest nonlinear model equation for diffusive waves. These nonlinear wave phenomena frequently appear in many domains of physics such as fluid dynamics [12]. This quasilinear parabolic equation is nonlinear parts of the advection-diffusion equation. The nonlinear advection-diffusion type equation is one of the popular and important models describing many phenomena derived from various areas of mathematical physics and engineering fields such as gas dynamics, hydrodynamics, shock waves[3], heat conduction [3], [23]. The first-order nonlinear parabolic partial differential equations model is nonlinear waves and which arise in gas dynamics, water waves, electrodynamics, chemical reactions, transport of pollutants flood, and ecological systems [8]. The nonlinear advection-diffusion type of equation represents the Burgers equation, the heat conduction equation [23], the nonlinear Schrödinger equation, the Navier-Stokes equation [3]. The Burgers equation is non linear partial differential equation of the conservation laws [8]. Burgers' equation is a famous nonlinear evolution equation [1] that was introduced by Burgers in 1948 [9] during his work on the theory of turbulence. But first, it was derived by Bateman [17] in 1915 during his work on the motion of fluids. It has interesting properties of nonlinear advection [8]. The Burgers equation belongs to the class of Navier-Stokes equation [5], [7] is a fundamental partial differential equation from fluid mechanics [7]. Therefore, studying the solution of the Burgers equation will be helpful to solve the Navier-Stokes equations [8]. This equation can also be considered as a simplified form of the Navier-Stokes equation [5], [23] due to the form of the nonlinear convection term and the occurrence of a viscosity term [11]. Nonlinear Burger equation occurs in various areas of applied mathematics and physics, such as shock waves [1],[16], dispersive water [16], turbulence [1, 4], modeling of gas dynamics, traffic flow, heat conduction [1], [15], viscous flow [1], acoustics [2], and continuous stochastic processes [14].

First-order quasilinear parabolic partial differential equation plays a crucial role in applied mathematics and physics. The importance of obtaining the exact or approximate solutions for these PDEs in physics and mathematics is still a hot topic research area. For that purpose, different methods have been put forward for seeking various

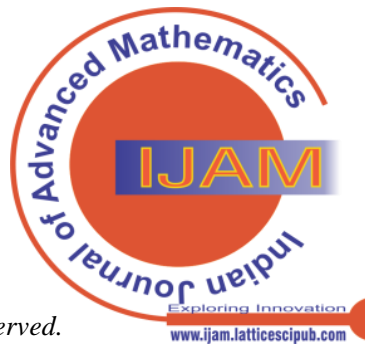


exact and numerical solutions of multifarious physical models described using these nonlinear PDEs. A well-known model was first introduced by Bateman [17], who found its steady solutions, descriptive of certain viscous flows.

It was later proposed by Burgers [9] as one of a class of equations describing mathematical models of turbulence. Khater et al. [12] proposed the Chebyshev spectral collocation method for solving the coupled Burgers' equations. With pseudo-spectral methods care must be taken with the round-off error issue when higher derivatives or a large several points is involved. Gowrisankar, S., and Natesan, S. [7] present the numerical solution of singularly perturbed initial-boundary Burgers' equation by using an efficient robust numerical method. They provide an $e$-uniformly convergent numerical method for the singularly perturbed Burger equation. They obtain uniform convergence concerning the perturbation parameter $\varepsilon$. Even though the method is capable of approximating Burger's equation, they failed to solve a relatively small perturbation parameter. Amit [6] Present solution of burger equation using seventh order convergent weakly $L$-stable Newton Cotes formula and application of Burger's equation. They used Hermit's interpolation polynomial approximation (oscillatory interpolation) and explicit backward Taylor's polynomial approximation. Both Hermits interpolation and Taylor series method is computationally time-consuming for a large number of the grid point. So the solution is containing high contaminated round-off error and truncation error. Solomon et al. [3] presented a solution of the nonlinear advection-diffusion equation by a backward semi-Lagrangian method. The method is computationally difficult for a large number of grid points. Sachin [8] presents the numerical solution of the burger equation by using the Crank-Nicolson type method. This method is of computational cost because it requires a large number of grid points to give an accurate solution for the Burger equation. Reza Abazaria and Borhanifar [22] presented the solution of Burgers and coupled Burgers' equation by using the differential transform method (DTM). DTM is a semi-numerical-analytic technique that formalizes the Taylor series differently. The Taylor series method is computationally time-consuming for large orders and high contaminated round-off error and truncation error. Jie Zhao et. al. [1] presents the numerical solution of the burger equation by using the Mixed Finite Volume Element Methods. This method is the high computational cost for a large number of the grid point. It means that as space size is decreasing, the complication of the method is increasing. So the accuracy of the solution is decreased. It was also difficult to apply this method to the complex solution domain.

However, still, the accuracy of the method needs attention; because the treatment of the method used to solve the nonlinear parabolic partial differential equation is not trivial distribution. Even though the accuracy of the aforementioned methods needs attention, they require large memory and long computational cost. So the treatments of this method present severe difficulties that have to be addressed to ensure the accuracy of the solution. To this end, this paper aims to present an "Average Based Differential Quadrature Method along spatial direction and central finite difference scheme in the direction of temporal variable" that is capable of solving "One-Dimensional Homogeneous First Order Nonlinear Parabolic Equation which is called Burger equation" and approximate the exact solution. The fully discretization this present method leads the system of linear equations which is solved by using LU-matrix decomposition method. The convergence has been shown in the sense of $L_{\infty}$ norm and

$L_{2}$ norm so that the local behavior of the solution is captured exactly. The stability and convergence of the present method are also investigated (analyzed).

\section{Statement of the problem}

Consider that the following homogenous nonlinear parabolic partial differential equation which is called $1 \mathrm{D}$ Burger equation considered in [1] given by:

$$
\begin{gathered}
U_{t}+a U U_{x}-v U_{x x}=0, \\
(x, t) \in(\mathrm{a}, \mathrm{b}) \times[0, \mathrm{~T})
\end{gathered}
$$

with initial and boundary condition respectively

$$
\begin{gathered}
U(x, 0)=U_{0}(x), \quad a \leq x \leq b, \\
U(a, t)=U(b, t)=0,0 \leq t \leq T
\end{gathered}
$$

where $\mathrm{v}>0$ is the coefficient of kinematic viscosity, $\mathrm{a}>0$ is diffusion coefficient, $0<T<\infty$ and $u_{0}(x)$ assumed to be sufficiently smooth functions for the existence and the

uniqueness of the solution [20], [24]. The solution A may represent a temperature for heat transfer or a species concentration for mass transfer at position $\mathrm{x}$ and time $\mathrm{t}$ with the advection velocity $u$ [3]. Now we define a mesh size $h$ and $\mathrm{k}$ and the constant grid point by drawing equidistance horizontal and vertical line of distance $h$ and $k$ respectively in $h$ and $k$ ' direction. These lines are called gridlines and the point at which they interacting are known as the mesh point. The mesh point that lies at end of the domain is called the boundary point. The points that lie inside the region are called interiors points. The goal is to approximate the solution ' $\mathrm{u}_{\mathrm{j} \mathrm{n}}{ }^{\mathrm{a}}$ at the interior mesh points. Hence we discretized the solution domain as:

$$
\begin{aligned}
& a=x_{0}<x_{1}<x_{2}<\cdots<x_{M}=b \\
& 0=t_{0}<t_{1}<t_{2}<\cdots<t_{N}=T
\end{aligned}
$$

where $x_{j+1}=x_{j}+j h$ and $t_{n+1}=t_{n}+n k$, $j=0(1) M, n=0(1) N . M$ and $N$ are the maximum numbers of grid points respectively in the $\mathrm{x}$ and $\mathrm{t}$ direction. Then the present paper is organized as follows. Section two is a preliminary, Section three is a description of numerical methods, Section four is existence of solution, Section five is Stability and convergence analysis, Section six is Results of numerical experiments. Section seven is Discussion and Section eight is the conclusion.

\section{PRELIMINARIES}

\subsection{Differential Quadrature Method}

The differential quadrature method is one of the most efficient numerical methods to solve partial differential equations. 
Different differential quadrature methods were developed by Bellman R. and Castiin in 1971 as cited in [25].

A variety of methods have been developed based on the DQ method, including Radial basis based differential quadrature methods [28], the polynomial-based differential quadrature (PDQ) and the Fourier-expansion-based differential quadrature methods (FDQ) [25]. The basic idea behind the DQ method is to determine the weighting coefficients for any order derivatives by using a weighted sum of functional values at a set of selected grid points [26]. PDQ and FDQM are highly efficient methods by using a small number of grid points, they are not efficient when the number of grid points is large and they are also sensitive to grid point distribution. While the PDQ and FDQ methods can obtain accurate results using only a small number of grid points, they are mesh-based methods [28], [30]. Hence in particularly we use Lagrange interpolation polynomial basis for polynomial-based differential quadrature method to discretize partial derivative involving with spatial variable associated with a weighted average and obtain numerical scheme for present works. These Lagrange interpolation polynomial bases given by:

$\varphi_{p}(x)=\frac{\widetilde{\Pi}(x)}{\left(x-x_{p}\right) \Pi^{[\mathbb{I}]}(x)}$

where $p=1(1) M$ and the denominator and nominator product function for this basis defined on the specific grid point defined in Eq.(3) are given by: $\bar{\Pi}(x)=\left(x-x_{1}\right)\left(x-x_{2}\right)\left(x-x_{3}\right) \ldots\left(x-x_{M}\right)$

$$
\begin{aligned}
& \Pi^{(1)}(x)=\left(x_{j}-x_{1}\right)\left(x_{j}-x_{2}\right) \ldots\left(x_{j}-x_{j-1}\right)\left(x_{j}-\right. \\
& \left.x_{j+1}\right) \ldots\left(x-x_{M}\right)\left(x-x_{M}\right)
\end{aligned}
$$

\subsection{Weighted average scheme}

We have now considered two finite difference methods, which differ only in that one approximates the second space derivative by three points on the old time level, $t_{n}$, and the other uses the three points on the new time level, $t_{n+1}$ [32]. A natural generalization is to an approximation which uses all six of these points. This can be regarded as taking a weighted average of the two formulae. See [23], [32],[33].

\section{NUMERICAL METHODS}

\subsection{The Temporal discretization}

Assuming that $u(x, t)$ has continuous higher order partial derivative on the region $[a, b] x[0, T]$. . By using Taylor series expansion, we have:

$$
\begin{aligned}
& u_{j n+1}=u_{j n}+k \partial_{t} u_{j n}+\frac{k^{2}}{2 !} \partial_{t}^{2} u_{j n}+\cdots \\
& u_{j n-1}=u_{j n}-k \partial_{t} u_{j n}+\frac{k^{2}}{2 !} \partial_{t}^{2} u_{j n}+\cdots
\end{aligned}
$$

Subtract the second equation from the first equation, the central difference equation of derivative of displacement function concerning temporal variable at the point $\left(x_{j}, t_{n}\right)$ :

$$
\partial_{t} U_{j n}=\frac{U_{j n+1}-U_{j n-1}}{2 k}+T_{2}
$$

Where $T_{1}=-\frac{k^{2}}{6} \partial_{t}^{3} u_{j n}$ local truncation error is the term for the discretization of temporal derivative. Now cutting this local truncation error from Eq. (6) and substituting the result to given PDEs in Eq. (1) associated with the weighted average in time of remaining terms we obtain the semi-discretization of the form: $\frac{U_{n+1}-U_{n-1}}{2 k}=\theta\left[a U U_{x}-v U_{x x}\right]_{n+1}+$ $(1-\theta)\left[a U U_{x}-v U_{x x}\right]_{n-1}$ $U_{n+1}-U_{n-1}=2 k\left[\theta\left[a U U_{x}-v U_{w}\right]_{n+1}+(1-\theta)\left[a U U_{w}-v U_{w}\right]_{n-1}\right]$ $U_{n+1}-2 k \theta\left[a U U_{n}-v U_{w}\right]_{n+1}=U_{n-1}+(1-\theta)\left[a U U_{n}-V U_{w}\right]_{n-1}$

$$
\begin{gathered}
v 2 k \theta\left(U_{n+1}\right)_{x x}+\left[1-v 2 k \theta a\left(U_{n+1}\right)_{x}\right] U_{n+1} \\
=\left[1+v 2 k(1-\theta) a\left(U_{n}\right)_{x}\right] U_{n-1}=v 2 k(1-\theta)\left(U_{n-1}\right)_{x x} \\
U_{n+1}(a)=0, U_{n+1}(b)=0,0 \leq n \leq N-1 \\
U(x, 0)=U_{0}(x)
\end{gathered}
$$

where $0 \leq \theta \leq 1$ and $U_{n+1}$ is the solution of the above differential equation at the $(n+1)^{t h}$ time step. Since Eq.(7) is a non-linear system of difference equation. So we linearize the non-linear problem in Eq. (7) by using the quasilinearization process which yields the following difference equation. For the initial condition of the above equation $U_{0}(x)=g(x)$, by quasilinearization process, this problem becomes:

$$
\begin{aligned}
& 2 k \theta v\left(U_{n+1}^{(i+1)}\right)_{x x}+2 k \theta v U_{n+1}^{(i)}\left(U_{n+1}^{(i+1)}\right)_{x}+ \\
& {\left[1-v 2 k \theta a\left(U_{n+1}^{(i)}\right)_{x}\right] U_{n+1}^{(i+1)}=} \\
& \left.U_{n+1}^{(i)}+4 k \theta v v_{n+1}^{(i)}\left(v_{n+1}^{(i)}\right)_{x}+-v 2 k(1-\theta)\left(U_{n-1}^{(i+1)}\right)_{x=}+\left[1+v 2 k(1-\theta) a\left(v_{n-1}^{(i)}\right)\right]_{x}\right]_{n-1}^{(i+1)}
\end{aligned}
$$

with the boundary and initials condition respectively

$$
\begin{aligned}
& U_{n+1}^{(i+1)}(a)=0, U_{n+1}^{(i+1)}(b)=0, \quad 0 \leq n \leq N-1 \\
& U_{0}^{(i+1)}=g(x)
\end{aligned}
$$

where initial guess $u^{(0)}$ and $i$ is the iteration index. Let $U^{(i+1)}=V$ in the above equation we obtain

$$
\begin{aligned}
& 2 k \theta v\left(V_{n+1}\right)_{x x}+2 k \theta v U_{n+1}^{(i)}\left(V_{n+1}\right)_{x}+ \\
& {\left[1-v 2 k \theta a\left(U_{n+1}^{(i)}\right)\right] V_{n+1}=} \\
& U_{n+1}^{(i)}+4 k \theta v U_{n+1}^{(i)}\left(U_{n+1}^{(i)}\right)_{x}+v 2 k(1-\theta)\left(V_{n-1}\right)_{x x}+\left[1+v 2 k(1-\theta) a\left(U_{n}^{(i)}\right)_{x}\right]_{n-1}
\end{aligned}
$$

with the boundary and initials condition respectively

$$
\begin{aligned}
& V_{n+1}(a)=0, V_{n+1}(b)=0,0 \leq n \leq N-1 \\
& V_{0}=g(x)
\end{aligned}
$$


For the sake of simplicity, dividing both side of Eq.(9) by $2 k$ and by letting

$$
\begin{aligned}
& {\left[\frac{1}{2 k}-v \theta a\left(U_{n+1}^{(i)}\right)_{x}\right]=d^{(i)}(x), U_{n+1}^{(i)}=b^{(i)}(x)} \\
& {\left[\frac{1}{2 k}+2 \theta v\left(U_{n+1}^{(i)}\right)_{x}\right] U_{n+1}^{(i)}+v(1-\theta)\left(V_{n-1}\right)_{x x}+} \\
& {\left[\frac{1}{2 k}+v(1-\theta) a\left(U_{n}^{(i)}\right)_{x}\right] V_{n}=H^{(i)}(x)}
\end{aligned}
$$

Now substituting Eq. (10) into Eq. (9) we obtain: $\theta v\left(V_{n+1}\right)_{x x}+\theta b^{(i)}(x)\left(V_{n+1}(x)\right)_{x}+$ $d^{(i)}(x) V_{n+1}(x)=H^{(i)}(x)$ $V_{0}=g(x)$

Now let us consider that $b^{(i)}(x) \geq \beta>0$ and $d^{(i)}(x) \geq y>0$ for which $\beta$ and $\gamma$ are the lower boundaries of $b^{(i)}(x)$ and $d^{(i)}(x)$ respectively for all $a \leq x \leq b$. Hence Eq. (11) can be written as:

$$
D_{\theta} V_{n+1}(x)=H^{(i)}(x)
$$

with its initial and boundary conditions respectively

$$
\begin{aligned}
& V_{n+1}(a)=0, V_{n+1}(b)=0,0 \leq n \leq N-1 \\
& V_{0}=g(x)
\end{aligned}
$$

where $D_{\theta}$ is difference operator which is defined on difference scheme in Eq. (11) which is given by:

$D_{\theta} V_{n+1}(x)=\theta v\left(V_{n+1}\right)_{x x}+\theta v b^{(i)}(x)\left(V_{n+1}(x)\right)_{x}+d^{(i)}(x) V_{n+1}(x)$

\subsection{The spatial discretization}

From the primary idea of the differential quadrature method, $U_{x}(x)$ and $U_{x x}(x)$ in Eq. (1) can be expressed as a linear combination of functional values at each grid point. It is given

$U_{x}\left(x_{i}, t\right)=\sum_{j=0}^{M} W_{i j} U\left(x_{j}, t\right), \quad U_{x x}\left(x_{i}, t\right)=$

$\sum_{j=0}^{M} \tilde{W}_{i j} U\left(x_{j}, t\right)$

where $W_{i j}$ and $\widetilde{W}_{i j}$ represent the weighting coefficients $[25,26,27,28]$ for $i=1,2, \ldots, M$

Hence by using the basis function in Eq. (4), the weighting coefficients of the first and second-order partial are respectively calculated by as in [26, 27]:

$W_{i j}=\frac{\Pi\left(x_{i}\right)}{\left(x_{j}-x_{p}\right) \Pi^{(\mathbb{1})}\left(x_{j}\right)}$, for $i \neq j$ and

$W_{i i}=-\sum_{j=1}^{M} W_{i j}, i=1(1) M$.

$i \neq j$

$\widetilde{W}_{i j}=2 W_{i j}\left(W_{i j}-\frac{1}{\left(x_{i}-x_{j}\right)}\right), i, j=1(1) M_{v} i \neq j$

and $\quad \tilde{W}_{i i}=-\sum_{\substack{j=1 \\ i \neq j}}^{M} \tilde{W}_{i j}, i=1(1) M$.

Since we assuming $\boldsymbol{U}^{(i+1)}=\boldsymbol{V}$.Thus using this obtained weighting coefficient and substituting Eq.(14) into Eq.(11), the full discretization of given non-linear parabolic partial differential equation

in Eq.(1)

$$
\begin{aligned}
& \theta v \sum_{p=1}^{M} \tilde{W}_{j p} V_{n+1, p}+\theta b^{(i)} \sum_{p=1}^{M} W_{j p} V_{n+1_{p} p}+ \\
& d^{(i)} V_{n+1, j}=H^{(i)}\left(x_{j}\right) \\
& V_{0}=g\left(x_{j}\right) \\
& V_{n+1,0}=0, V_{n+1_{2} M}=0
\end{aligned}
$$

Thus using the discrete scheme in Eq.(15), we obtain the desired solution for non-linear parabolic partial differential equation in Eq.(1).

\section{EXISTENCE OF UNIQUE AND CONTINUOUS SOLUTION}

The following lemmas [18], [29] are necessary for the existence and uniqueness of the solution and for the problem to be well-posed.

Lemma 1. Maximum principle [18]. Assume that any function $f \in C^{2}(a, b)$ satisfies $f(a) \geq 0$ and $f(b) \geq 0$. Then, $D_{\theta} f(x) \geq 0$ for all $x \in(a, b)$ implies that $f(x) \geq 0$ for all $x \in(a, b)$. Proof:

Let $(a, b)=(0,1)$ and $\xi^{*}$ be any number such that $f\left(\xi^{*}\right)=\min _{0 \leq x \leq 1} f(x)$ and suppose that $f\left(\xi^{*}\right)<0$. It is clear that $\xi^{*} \in(0,1)$.Therefore we have $f_{x}\left(\xi^{*}\right)=0 \quad, \quad f_{x x}\left(\xi^{*}\right) \geq 0 \quad$ and $D_{\theta} f\left(\xi^{*}\right)=\theta v f_{x x}\left(\xi^{*}\right)+\theta v b^{(i)}\left(\xi^{*}\right) f_{x}\left(\xi^{*}\right)+d^{(i)}\left(\xi^{*}\right) f\left(\xi^{*}\right.$ Since $d^{(i)}(x) \geq 0$ for all $x \in(0,1)$. Therefore $D_{\theta} f\left(\xi^{*}\right)<0$, which contradicts the assumption, therefore it follows that $f(x) \geq 0$. Hence $f(x) \geq 0$ for all $x \in(0,1)$.

Lemma 2. [23], [29] Let $V_{n+1}(x)$ be the smooth continuous solution of Eq. (11), there exists a constant $C$ such that

$$
\begin{aligned}
& \qquad \begin{array}{l}
\left\|V_{n+1}(x)\right\|<C \text { For all } x \in(0,1) \text { and } \\
\left\|V_{n+1}(x)\right\| \leq C\left(1+G^{-p} e^{\alpha(1-x) / G}\right)
\end{array} \\
& \text { For all } x \in(0,1), p=1,2,3,4, \ldots M
\end{aligned}
$$

Proof:

Defining the barrier function $f^{ \pm}(x)=C(1+x)+V_{n+1}(x)$ such that $f^{ \pm}(x) \geq 0$, $f^{ \pm}(1) \geq 0$ and $\mathrm{C}$ are constant sufficiently become large. That $D_{\theta} f^{ \pm}(x) \geq 0$ for $x \in(0,1)$, using maximum principle for $f^{ \pm}(x)=0$ we have:

$$
V_{n+1}(x) \leq c(1+x) \text { For } x \in(0,1) \text {. }
$$

Hence $\left\|V_{n+1}(x)\right\|<C$ for all $x \in(0,1)$. Now, we find the bound of the derivatives of $V_{n+1}$ by the use of induction. Theorem 2 in [21] can be extended for $p=1,2,3, \ldots M$. So, the proof is similar to the prove in ref. [21].

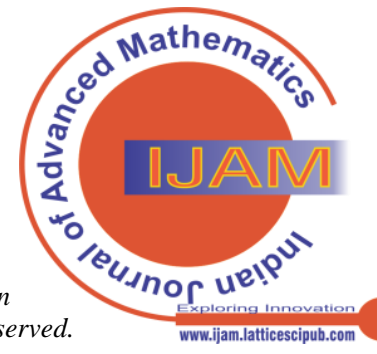


Lemma 3. [29](Continuous minimum principle). Assume that any function $f \in C^{2}(a, b)$ satisfies $f(a) \geq 0$ and $f(b) \geq 0$. Then, $D_{\theta} f(x) \leq 0$ for all $x \in(a, b)$ implies that $f(x) \geq 0$ for all $x \in(a, b)$.

Proof: See (29).

The uniqueness of the solution is implied by this maximum and minimum principle. Its existence follows trivially (as for non linear problems, the uniqueness of the solution implies its existence). This principle is now applied to prove that the solution of Eqs. (1)- (2) is bounded.

\section{STABILITY AND CONVERGENCE ANALYSIS}

Lemma 4. [23] Under the assumption that all the coordinates are in the interval $h$ (i.e. uniform mesh with length h), and the $M^{\text {th }}$ order derivative of the function $V_{n+1}$ is bounded as in Lemma 2, which is given,by: $\|\widetilde{\Pi}(x)\| \leq M(M-1)(M-2) \ldots(M-m+$ 1) $h^{M-m}$

$$
\|\widetilde{\Pi}(x)\| \leq h^{M-m}
$$

Proof. Since we approximate the function $V_{n+1}$ in domain concerning with spatial variable by using Lagrange interpolation basis function in order to approximate its derivative Hence using this basis function in Eq. (4) and differentiate it concerning $\mathrm{x}$, we obtain

$\tilde{\Pi}^{()}(x)=\prod_{p=2}^{M}\left(x-x_{p}\right)+\prod_{\substack{p=1 \\ p \neq 2}}^{M}\left(x-x_{p}\right)+\cdots+\prod_{\substack{p=1 \\ p \neq M-1}}^{M}\left(x-x_{p}\right)+\prod_{p=1}^{M-1}\left(x-x_{p}\right)$

M terms and Appling matrix norm on both sides we obtain

$$
\begin{aligned}
& \begin{array}{l}
\left\|\tilde{\Pi}^{(0)}(x)\right\|=\| \prod_{p=2}^{M}\left(x-x_{p}\right)+\underset{\substack{p=1 \\
p \neq 2}}{M}\left(x-x_{p}\right) \\
\cdots+\prod_{\substack{p=1 \\
p \neq M-1}}^{M}\left(x-x_{p}\right)+\prod_{p=1}^{M-1}\left(x-x_{p}\right) \| \\
\leq\left\|\prod_{p=2}^{M}\left(x-x_{p}\right)\right\|+\left\|\prod_{\substack{p=1 \\
p \neq 2}}^{M}\left(x-x_{p}\right)\right\|+\cdots+
\end{array} \\
& \left\|\prod_{\substack{p \neq M-1 \\
p \neq 1}}^{M}\left(x-x_{p}\right)\right\|+\left\|\prod_{p=1}^{M-1}\left(x-x_{p}\right)\right\|
\end{aligned}
$$

Let $\psi=\frac{x-x_{j}}{\mathrm{R}}$ where $x_{j}=x_{1}+(j-1) h$. Similarly, we have $x_{i}=\psi+(j-i) h \quad$ for all $i=1(1) M$ and $j=1(1) M$.Now by using this expiration we have:

$\prod_{p=2}^{M}\left(x-x_{p}\right)=(\psi+(j-2)) h(\psi+$ $(j-3)) h \ldots(\psi-(N-j)) h=h^{M-1} \mathrm{Z}(\psi)$

Therefore by using the theory of Lagrange's Interpolation error estimate, we have

$$
\left\|\Pi_{p=2}^{M}\left(x-x_{p}\right)\right\| \leq \| h^{M-1} Z\left(\psi\|\leq\| h^{M-1} \|\right.
$$

Now substituting Eq. (18) into Eq. (17) we obtain:
$\left\|\widetilde{\Pi}^{(0)}(x)\right\| \leq h^{M-1}+h^{M-1}+\cdots+h^{M-1} \leq M h^{M-1}$

Similarly for the second derivative we have:

$\left\|\tilde{\Pi}^{(2)}(x)\right\| \leq M(M-1) h^{M-J}$

For $I^{\text {th }}$ derivative, by using induction we have:

$\left\|\tilde{\Pi}^{(2)}(x)\right\| \leq M(M-1)(M-2)(M-$

3) $\ldots(M-J+1) h^{M-I}$

Hence the derivative of $V_{n+1}$ and the error in the approximating of functions are bounded.

Lemma 5. If the error for the $p^{\text {th }}$ order derivative approximation is defined as

$$
\begin{gathered}
L_{D}^{(p)}\left(V_{n+1}\right)=\frac{\partial^{(p) V_{n+1}}}{\partial x^{(p)}}-\frac{\partial^{\left.(p) Q_{M} V_{n+1}\right)}}{\partial x^{(p)}}, \\
p=1(1) M
\end{gathered}
$$

Where $Q_{M} V_{n+1}$ is the approximation of $V_{n+1}$ a polynomial of degree $\mathrm{M}-1$, then

$$
L_{D}^{(p)} V_{n+1}(\tilde{x})=\frac{V_{n+1}(\tilde{\zeta}) \widetilde{\Pi}(x)}{M-p} \text { and }
$$

$\left\|L_{D}^{(p)}\left(V_{n+1}\right)\right\| \leq \frac{C\left(1+G^{-p} e^{\alpha(1-x) / G}\right) h^{M-p}}{(M-p) !}$

Proof: The prove this lemma 5 is in [23]

Theorem 1. [23] Let $V_{n+1}\left(x_{j}\right)$ be a solution of Eq.(10) and $\tilde{V}_{n+1}$ be a solution of the full discretized problem in Eq.(15) at $\left(\frac{t}{n}+1\right)^{t / n}$ in temporal direction, then :

$$
\begin{aligned}
& \left\|\tilde{V}_{n+1}-V_{n+1}\left(x_{j}\right)\right\|_{\frac{1}{n}} \leq
\end{aligned}
$$

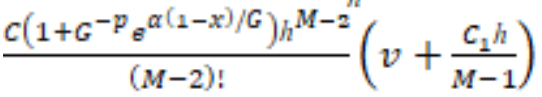

Where $\|.\|_{\frac{R}{3}}$ denote the discrete maximum norm.

Proof: Let $V_{n+1}\left(x_{j}\right)$ be a solution of Eq.(10) and $\tilde{V}_{n+1}$ be a solution of the full discretized problem in Eq.(15) at point $\left(x_{j}, t_{n+1}\right)$.Now subtracting Eq(11) from Eq.(15) the error term is:

$\tilde{V}_{n+1}-V_{n+1}\left(x_{j}\right)=\frac{1}{d^{(i)}\left(x_{j}\right)}\left(-v \theta L_{l h}^{(2)} V_{n+1}\left(x_{j}\right)+b^{(i)}\left(x_{j}\right) L_{h l}^{(1)} V_{n+1}\left(x_{j}\right)\right)$

Since $b^{(i)}$ and $d^{(i)}$ are a bounded sequence of the term. Now using this idea and Lemma 5 we have:

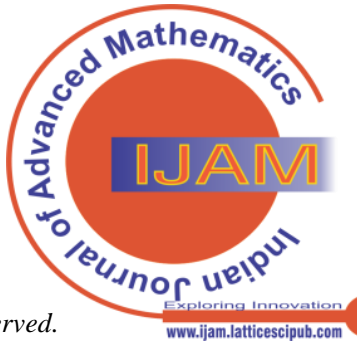


$\left\|\tilde{V}_{n+1}-V_{n+1}\left(x_{j}\right)\right\|_{\frac{\pi}{6}}=$

$\| \frac{1}{d^{(i)}\left(x_{j}\right)}\left(-v \theta L_{\frac{i}{i n}}^{(2)} V_{n+1}\left(x_{j}\right)+\right.$

$\left.b^{(i)}\left(x_{j}\right) L_{i}^{(1)} V_{n+1}\left(x_{j}\right)\right) \|_{i}$

$\leq$

$\frac{v \theta}{\| d^{(i)}\left(x_{j}\right)}\left\|L_{\frac{R}{2}}^{(2)} V_{n+1}\left(x_{j}\right)\right\|+$

$\theta\left|\frac{b^{(1)}\left(x_{j}\right)}{d^{(1)}\left(x_{j}\right)}\right|\left\|L_{i n}^{(1)} V_{n+1}\left(x_{j}\right)\right\|$

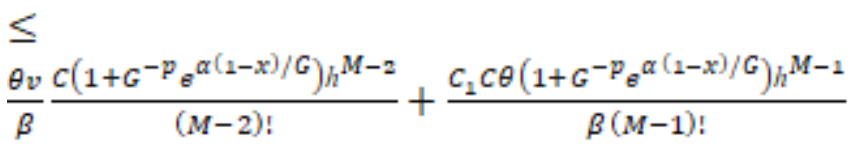

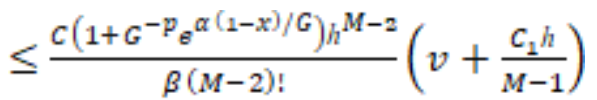

Corollary 1: Under the condition of the above theorem the proposed scheme is given in Eq. (15) is a well-posed scheme with the given initial and boundary condition.

Proof: Let $V\left(x_{j}, t_{n}\right)$ and $V_{n+1}$ are the exact and numerical solution of PDE in Eq.(1) at grid point $\left(x_{j}, t_{n}\right)$. Then the global error at the initial condition $\left(x_{j}, t_{0}\right)$ is defined by $E_{j, 0}=V\left(x_{j}, t_{0}\right)-V_{j 0}$. By using the condition of the above theorem 5 and Lemma 5. we have:

$\left\|E_{j, 0}\right\|=\left\|V\left(x_{j}, t_{0}\right)-V_{j 0}\right\|=C$

Hence the scheme is well-posed at $\left(x_{j}, t_{0}\right)$ for the initial condition. For $j \neq 0$ and $n=1(1) N$, Then the global error is

$$
\begin{aligned}
& \left\|E_{j n}\right\|=\left\|V\left(x_{j}, t_{j}\right)-V_{j n}\right\| \leq \\
& C \frac{\left(1+G^{-p} e^{\alpha(1-x) / G}\right) \mu^{M-2}}{\beta(M-2) !}\left(v+\frac{c_{1}{ }^{2}}{M-1}\right)
\end{aligned}
$$

Hence $\left\|E_{j n n}\right\| \rightarrow 0$ and bounded, Thus scheme is stable. Thus it is well-posed. So it is convergent.

\section{Criteria for Investigation the Accuracy of Proposed}

In this section, we investigate the accuracy of the present method. To show the accuracy of the present method, the Root Mean Square (RMS) error norm $\left(\mathrm{L}_{2}\right)$ and maximum absolute error norm $(\mathrm{L} \infty)$ are used to measure the accuracy of the proposed method. The RMS error and maximum absolute error are calculated as in the ref. [6] given by:

$$
\begin{gathered}
L_{2}=\sqrt{h \sum_{j=1}^{M}\left(\left|V\left(x_{j}, t_{n}\right)-V_{j n}\right|\right.} \\
L_{\infty}=\max _{1 \leq j \leq M}\left|V\left(x_{j}, t_{N}\right)-V_{j}\right|
\end{gathered}
$$

Where $V\left(x_{j}, t_{n}\right)$ and $V_{j n}$ are respectively exact and numerical solutions of the non linear parabolic equation at the grid point $\left(x_{j}, t_{n}\right)$

\section{RESULTS OF NUMERICAL EXPERIMENTS}

To test the validity of the proposed method, we have considered the following model problem.

Example 1. Consider that the following homogenous 1D non linear parabolic equation (Burger equation) considered in [1] given by:

$$
U_{t}+U U_{x}-v U_{x x}=0
$$

$$
(x, t) \in(0,1) \times[0,2)
$$

with initial and boundary condition respectively

$$
\begin{aligned}
& U(x, 0)=\sin \pi x \quad 0 \leq x \leq 1, \quad U(a, t)= \\
& U(b, t)=0,0 \leq t \leq 2
\end{aligned}
$$

The unique exact solution of the above Burger's equation is given by :

$$
\begin{aligned}
& U(x, t)=2 \pi v\left[\frac{\sum_{m=1}^{\infty} A_{m} e^{-v m^{2} \pi^{2} t} m \sin (m \pi x)}{A_{0}+\sum_{m=1}^{\infty} A_{m} e^{-v m^{2} \pi^{2} t} \cos (m \pi x)}\right] \\
& \text { where } \quad A_{0}=\int_{0}^{1} e^{\left(-\frac{1}{s \pi v}(1-\cos (\pi x))\right.} d x \quad \text { and } \\
& A_{m}=2 \int_{0}^{1} e^{\left(-\frac{1}{3 \pi v}(1-\cos (\pi x))\right) \cos (m \pi x)} d x \\
& m=1,2, \ldots n
\end{aligned}
$$

Example 2: Consider the in Eq. (1) on $[0,1]$ considered by Amit Kumar Verma et.al. in [6]

The unique exact solution of the above 1D non linear parabolic equation (Burger equation) is given by:

$$
U(x, t)=\pi v\left[\frac{e^{\left(-v^{2} \pi^{2} t / 4\right)} \sin (\pi x)+4 e^{\left(-v^{2} \pi^{2} t\right)} \sin (2 \pi x)}{4+e^{\left(-v^{2} \pi^{2} t / 4\right)} \cos (\pi x)+2 e^{\left(-v^{2} \pi^{2} t\right)} \cos (2 \pi x)}\right]
$$

Table 1 Comparison of maximum absolute error norm $L_{\infty \circ}$ and convergence orders for problem give in example one with computations carried out until final time $T=2$ with different mesh size.

\begin{tabular}{llllll}
\hline $\mathbf{h}$ & $\mathbf{k}$ & $\boldsymbol{L}_{\infty}$ & Order & $\boldsymbol{L}_{\infty}$ & Order \\
Present Method & & & & \\
$\mathbf{1} / \mathbf{2 0}$ & $1 / 20$ & $2.12402 \mathrm{E}-09$ & $\mathbf{0 . 9 2 3 6}$ & $5.95194 \mathrm{E}-09$ & $\mathbf{0 . 9 2 1 1 4}$ \\
$\mathbf{1} / \mathbf{4 0}$ & $1 / 40$ & $1.64798 \mathrm{E}-09$ & $\mathbf{0 . 9 2 3 9}$ & $1.61781 \mathrm{E}-09$ & $\mathbf{0 . 9 6 7 0 1}$ \\
$\mathbf{1} / \mathbf{8 0}$ & $1 / 80$ & $2.48655 \mathrm{E}-09$ & $\mathbf{0 . 9 3 8 7 6}$ & $2.56165 \mathrm{E}-10$ & $\mathbf{0 . 9 7 5 4}$ \\
$\mathbf{1} / \mathbf{1 6 0}$ & $1 / 160$ & $2.2706 \mathrm{E}-10$ & $\mathbf{0 . 9 3 9 9 1}$ & $3.74816 \mathrm{E}-10$ & $\mathbf{0 . 9 7 6 4 4}$ \\
\hline
\end{tabular}




\begin{tabular}{|c|c|c|c|c|c|}
\hline \multicolumn{6}{|c|}{ By Jie Z. et.al. in [1] } \\
\hline $1 / 20$ & $1 / 20$ & 2.6767E - 02 & & 2.1361E - 02 & \\
\hline $1 / 40$ & $1 / 40$ & 1.4241E - 02 & 0.9104 & 1.1424E - 02 & 0.9029 \\
\hline $1 / 80$ & $1 / 80$ & $7.4366 \mathrm{E}-03$ & 0.9374 & $5.9368 \mathrm{E}-03$ & 0.9444 \\
\hline $1 / 160$ & $1 / 160$ & $3.8548 E-03$ & 0.9480 & 3.0320E - 03 & 0.9694 \\
\hline
\end{tabular}

Fig. 1. Physical behaver numerical solution of Example 1 for $h=k=1 / 20$ and $v=1 / 48$

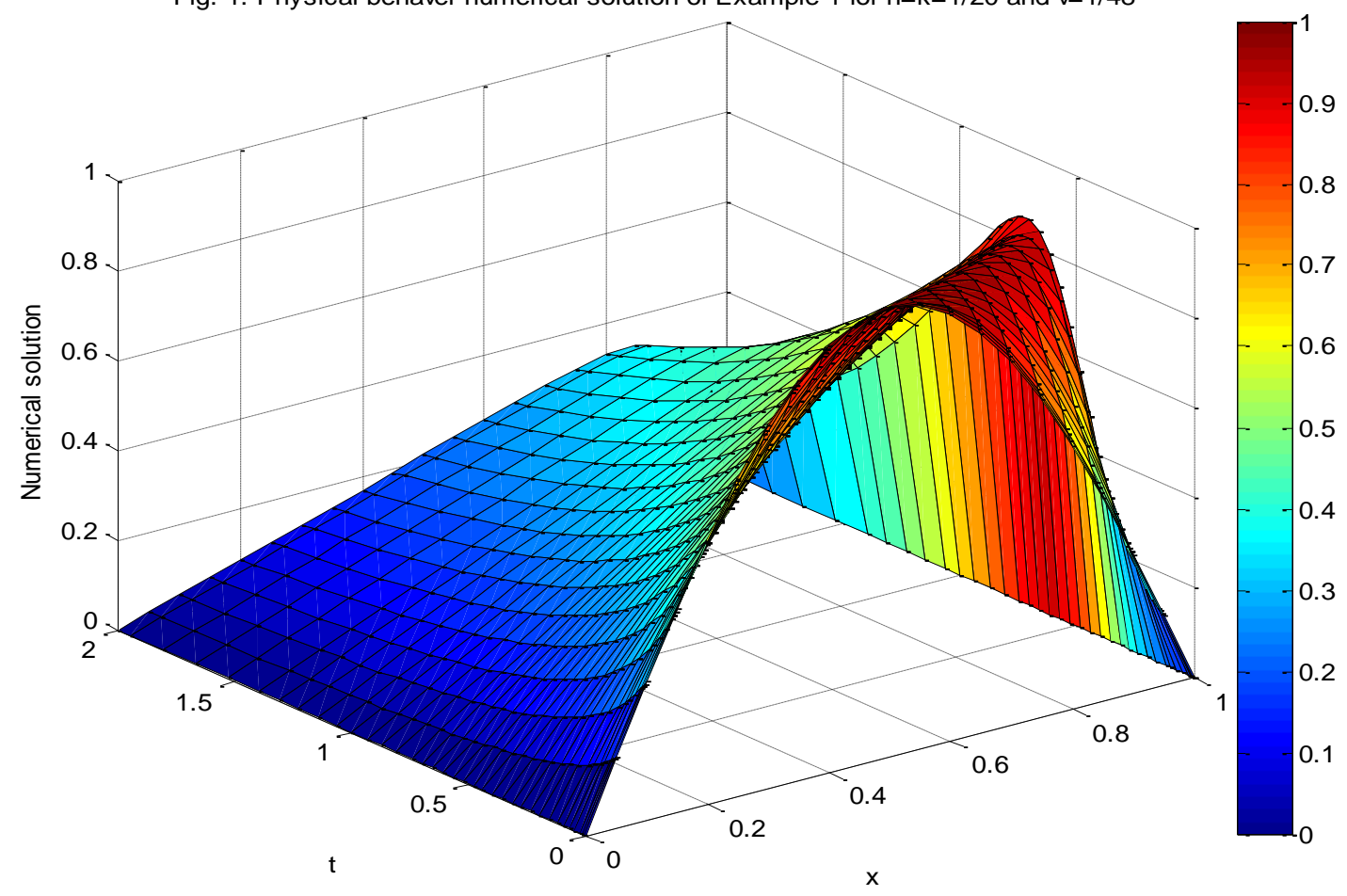

Fig 2.Physical behaver Exact solution of Example 1 for $h=k=1 / 20$ and $v=1 / 48$

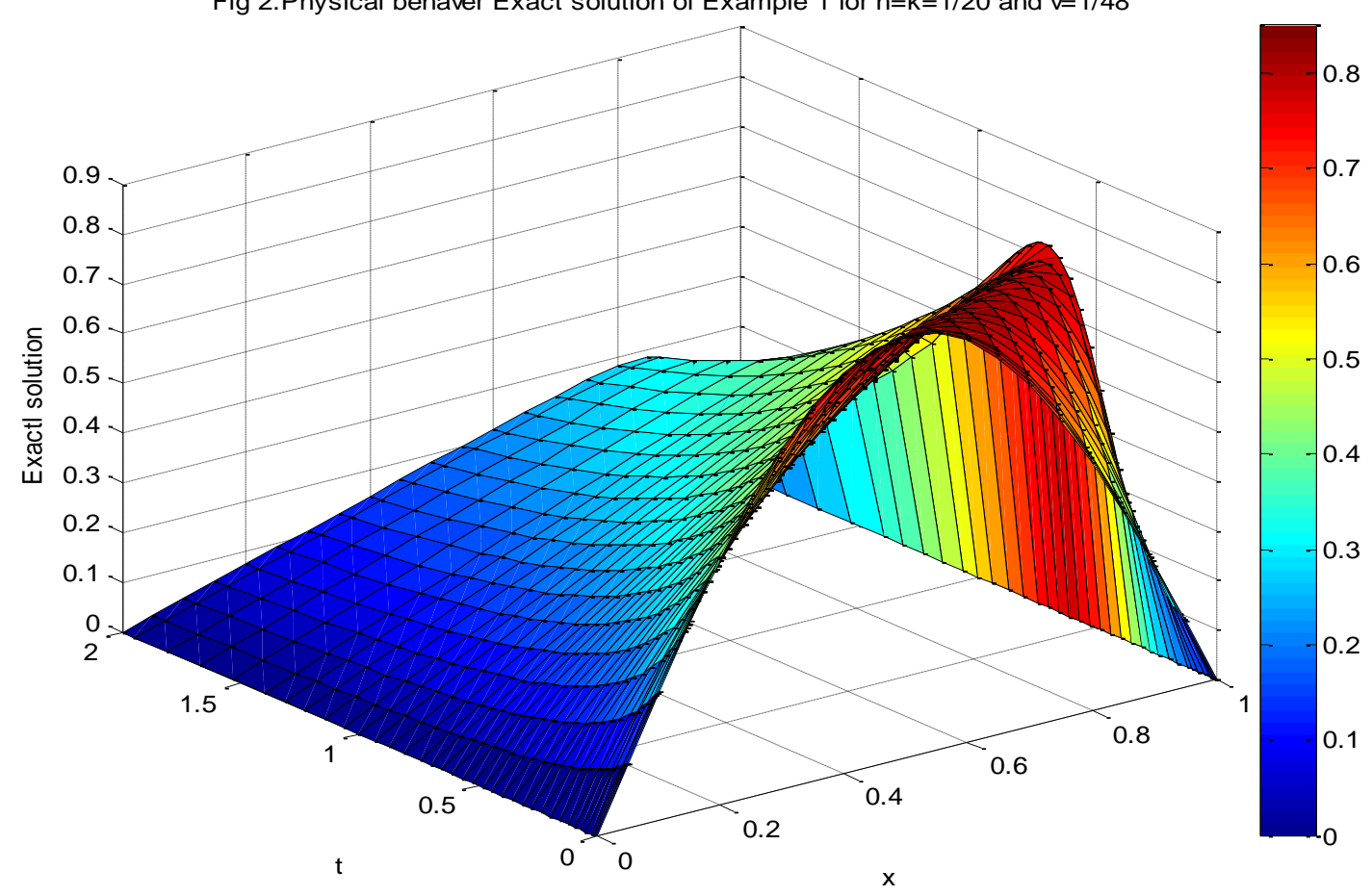




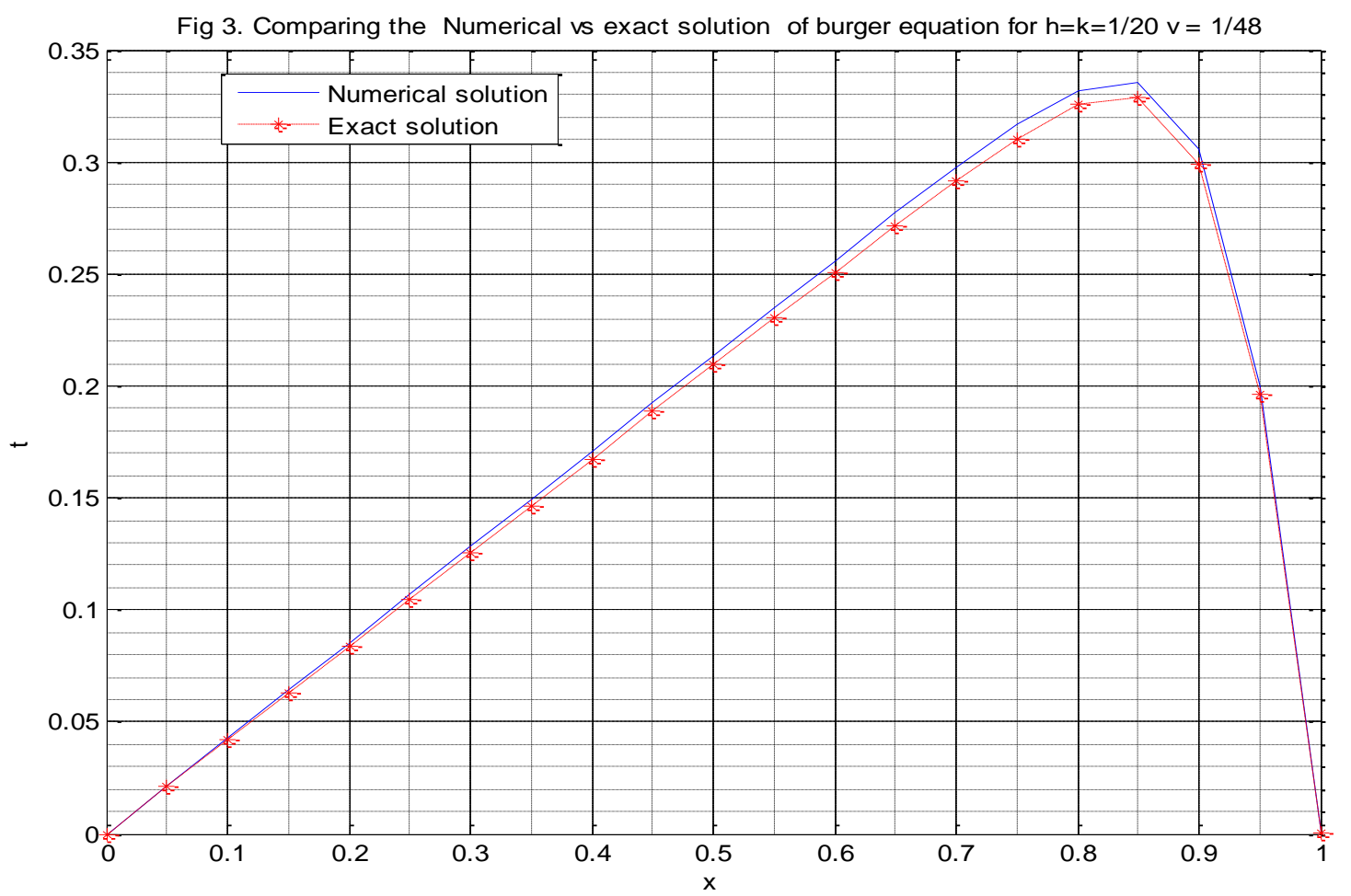

Fig 4. Maximum Absolute Error at each time step for $h=k=1 / 20$ and $v=1 / 48$

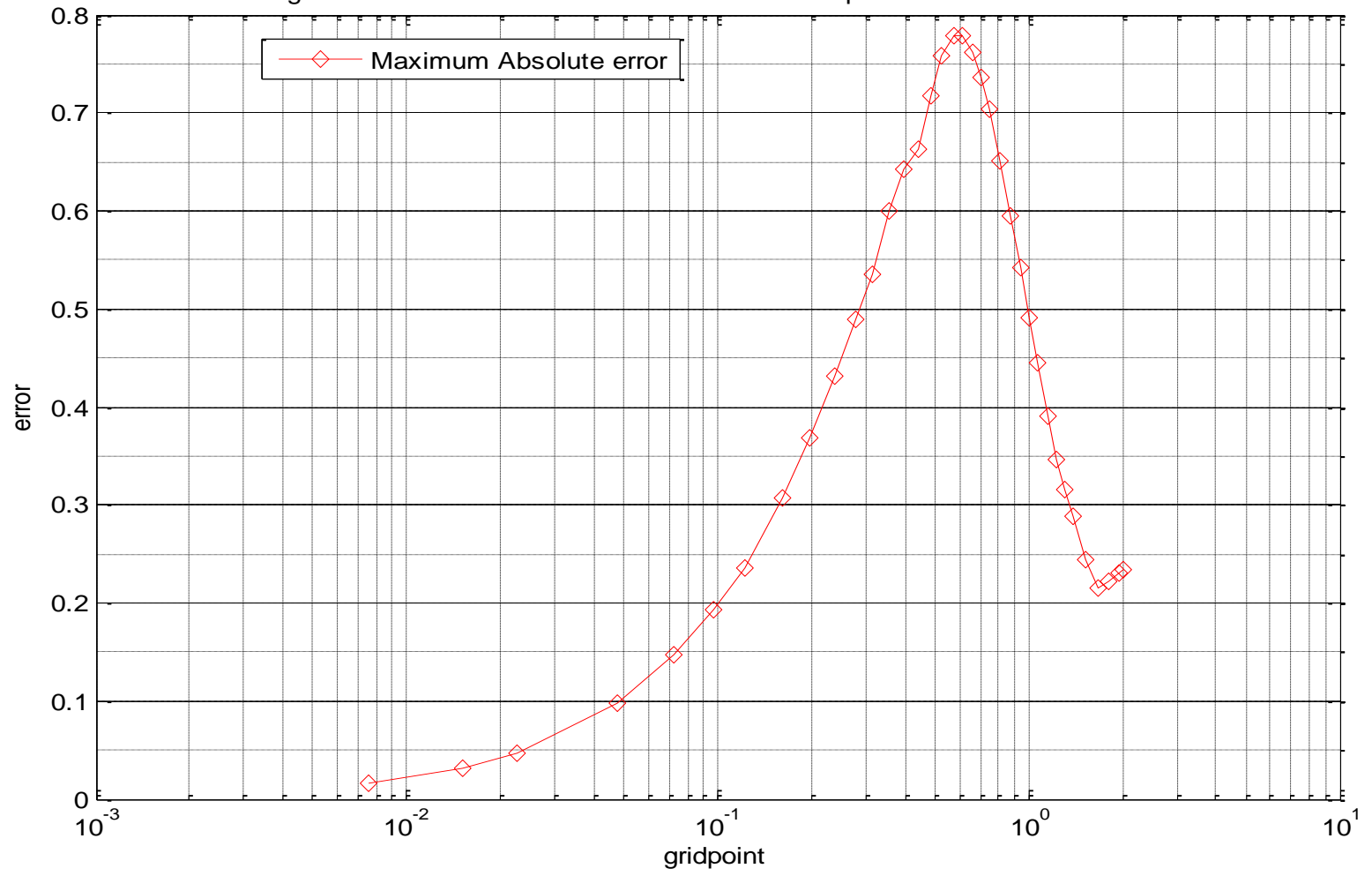


Fig. 5. Physical behaver numerical solution of Example 1 for $h=k=1 / 40$ and $v=1 / 48$

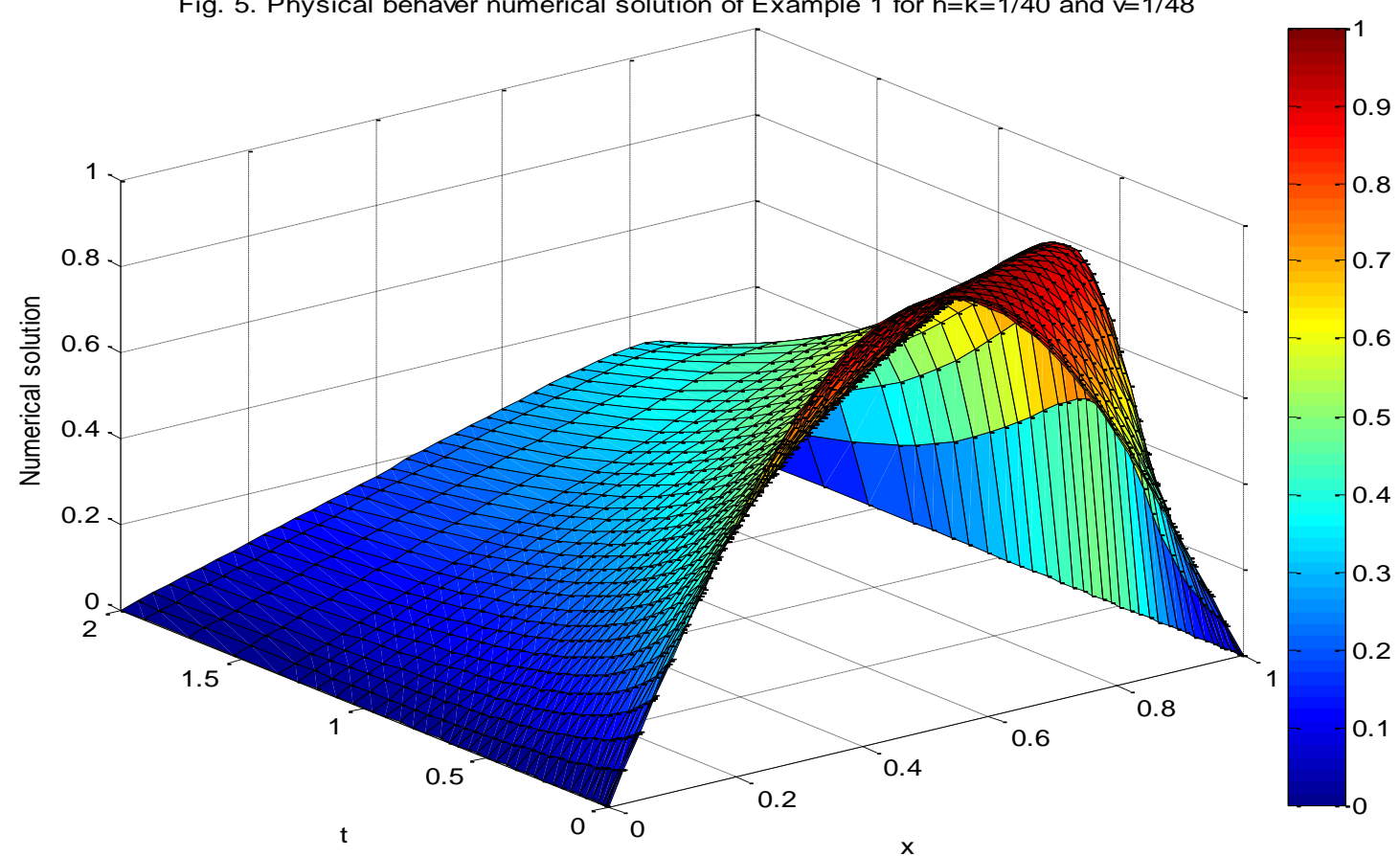

Fig 6.Physical behaver Exact solution of Example 1 for $h=k=1 / 40$ and $v=1 / 48$

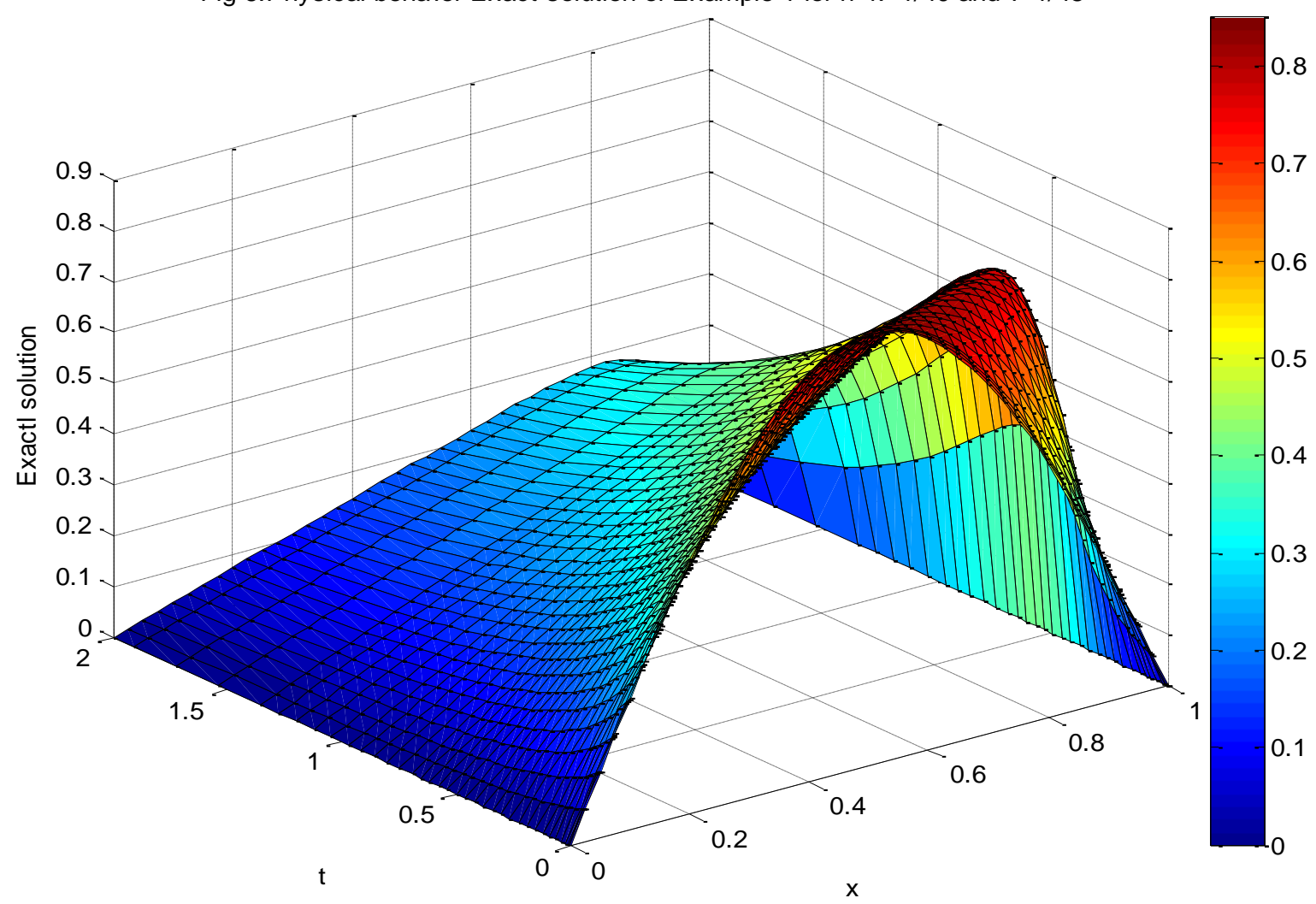



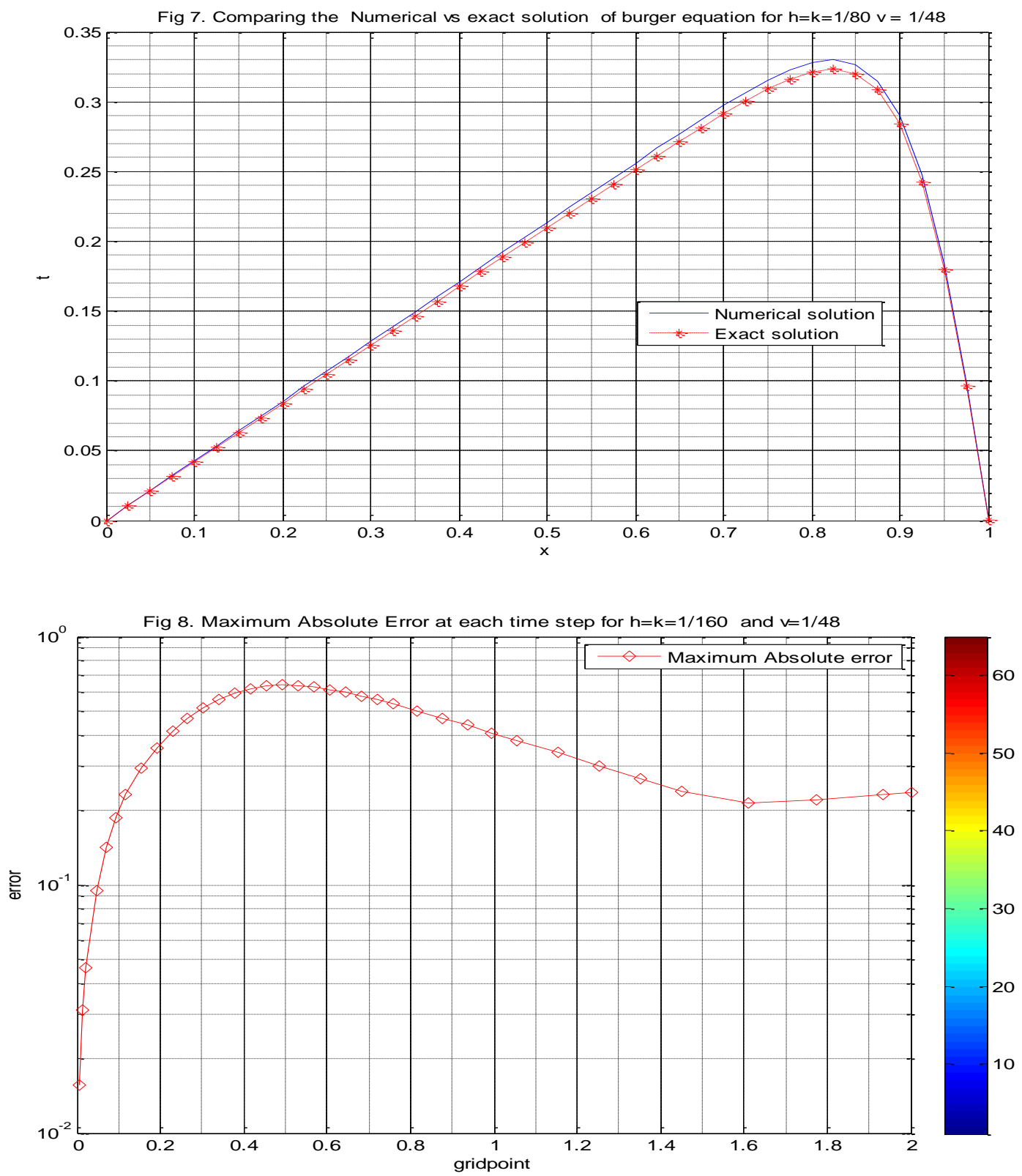

Table 2. Comparison of Root Mean Square (RMS) error norm $L_{2}$ and maximum absolute error norm $L_{\infty}$ for problem of example two with computations carried out until different final time and step-length , and fixed time step for $v=0.002$.

\begin{tabular}{llll}
\hline Mesh size & By SS Xie [29] By Amit in[11] & By present method
\end{tabular}

With computations carried out until final time $T=1.7$

\begin{tabular}{llllllll}
$\mathrm{h}$ & $\mathrm{k}$ & $\mathrm{L}_{\infty}$ & $\mathrm{L}_{2}$ & $\mathrm{~L}_{\infty}$ & $\mathrm{L}_{2}$ & $\mathrm{~L}_{\infty}$ & $\mathrm{L}_{2}$ \\
\hline 0.0005 & 0.01 & $2.9704 \mathrm{E}-02$ & $3.5936 \mathrm{E}-03$ & $5.02 \mathrm{E}-04$ & $1.67 \mathrm{E}-05$ & $3.2901 \mathrm{e}-11$ & $2.3613 \mathrm{e}-12$ \\
\hline 0.01 & 0.1 & & & & & $1.638 \mathrm{e}-012$ & $4.2081 \mathrm{e}-013$
\end{tabular}

With computations carried out until final time $T=3$

\begin{tabular}{|c|c|c|c|c|c|c|}
\hline 0.0005 & 1.9009E-02 & 2.635E-03 & $2.1289 \mathrm{E}-4$ & 8.14E-05 & $1.8124 \mathrm{e}-14$ & $6.2127 \mathrm{e}-15$ \\
\hline 0.01 & 0.01 & & & & $1.7182 \mathrm{e}-14$ & $5.9144 \mathrm{e}-15$ \\
\hline \multicolumn{7}{|c|}{ With computations carried out until final time $T=4$} \\
\hline 0.0005 & 0.01 & & & & $9.9107 e-15$ & $3.5461 \mathrm{e}-15$ \\
\hline 0.01 & 0.1 & & & & $9.4831 \mathrm{e}-15$ & $3.4040 \mathrm{e}-15$ \\
\hline
\end{tabular}

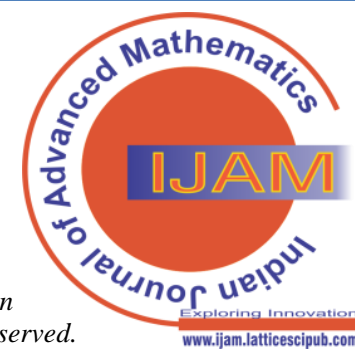


Fig. 9. Physical behaver numerical solution of Example 2 for $h=0.1, k=0.001$ and $v=0.002$

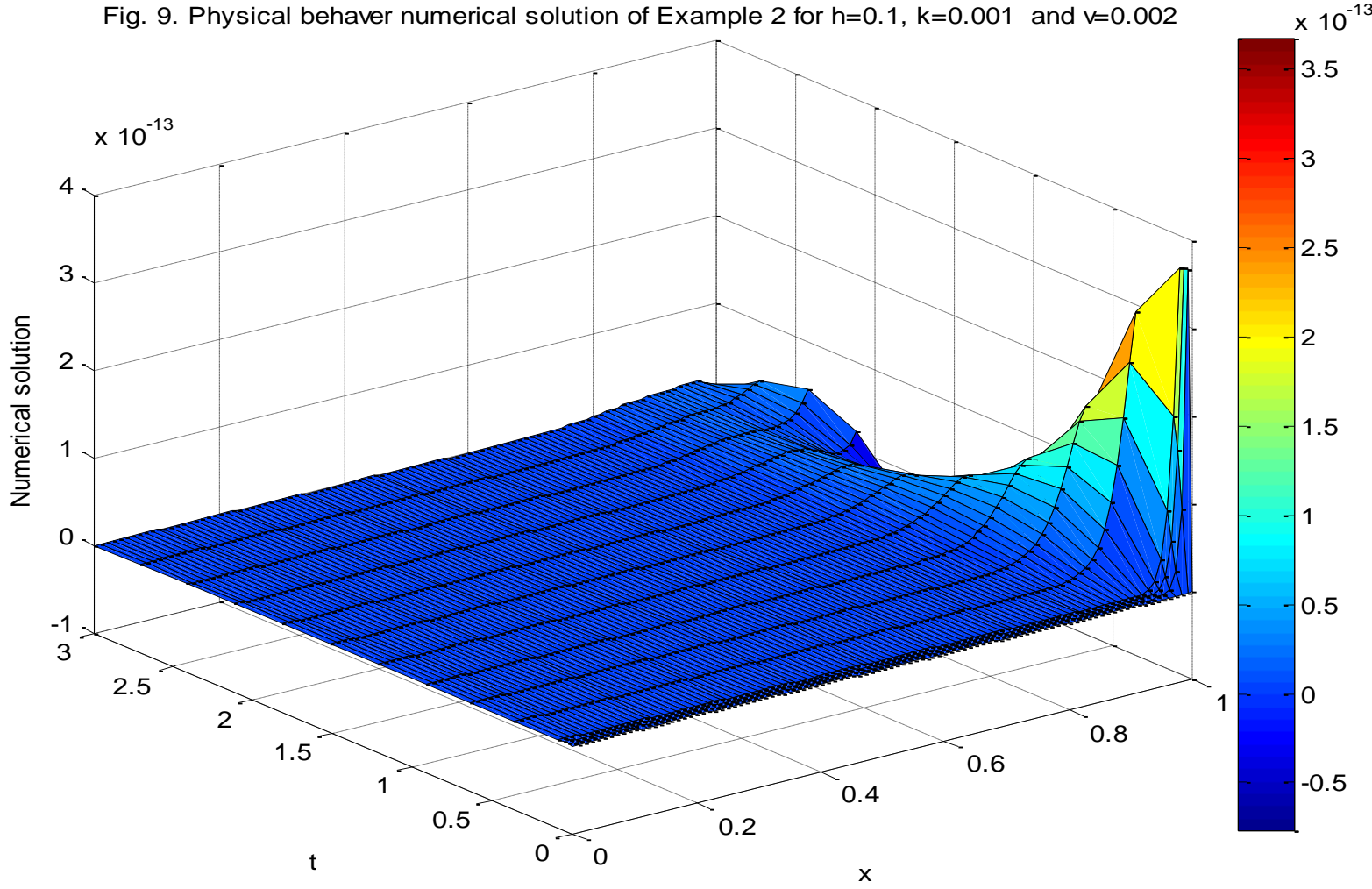

Fig 10.Physical behaver Exact solution of Example 2 for $h=0.1, k=0.001$ and $v=0.002$

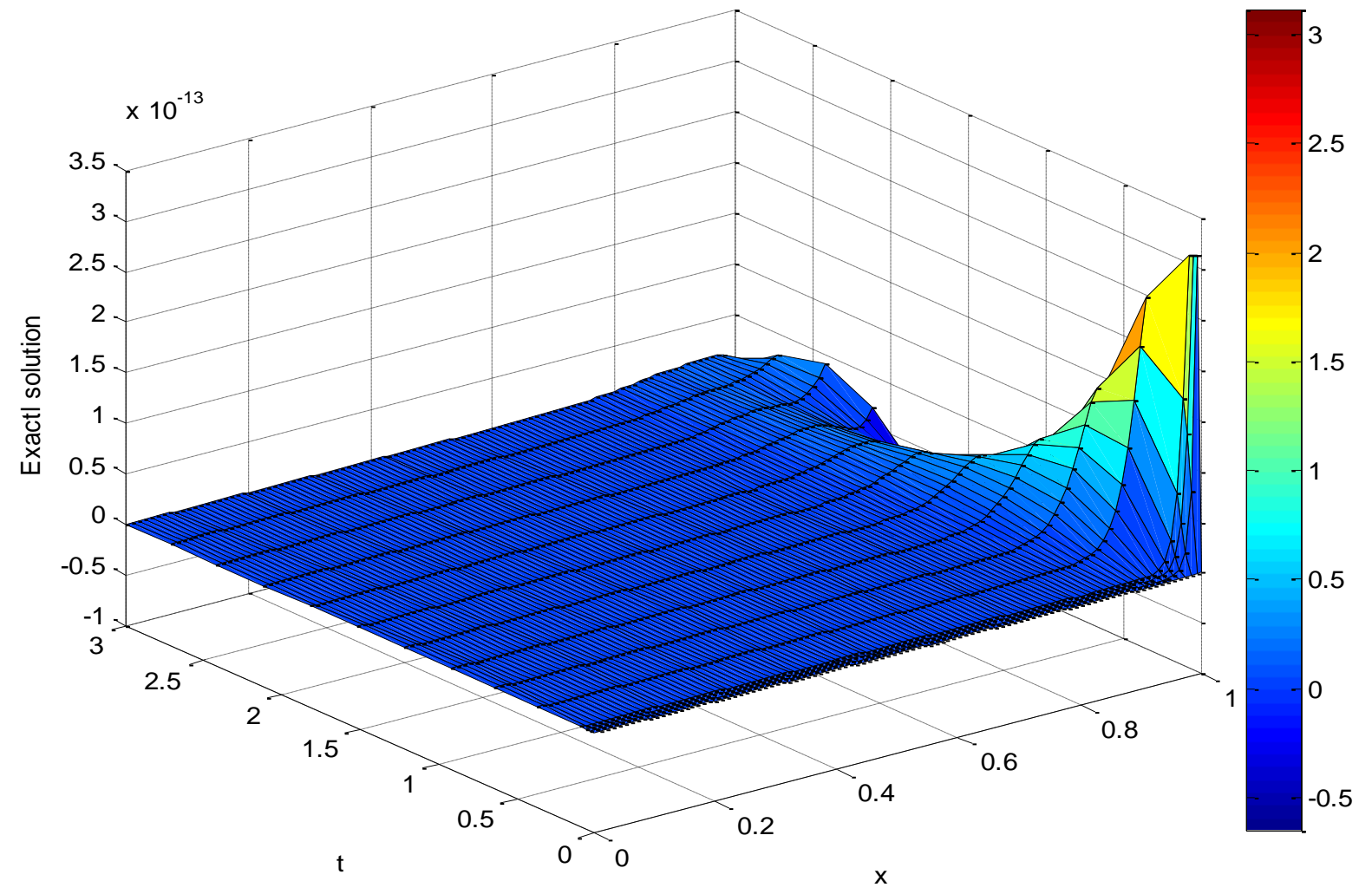



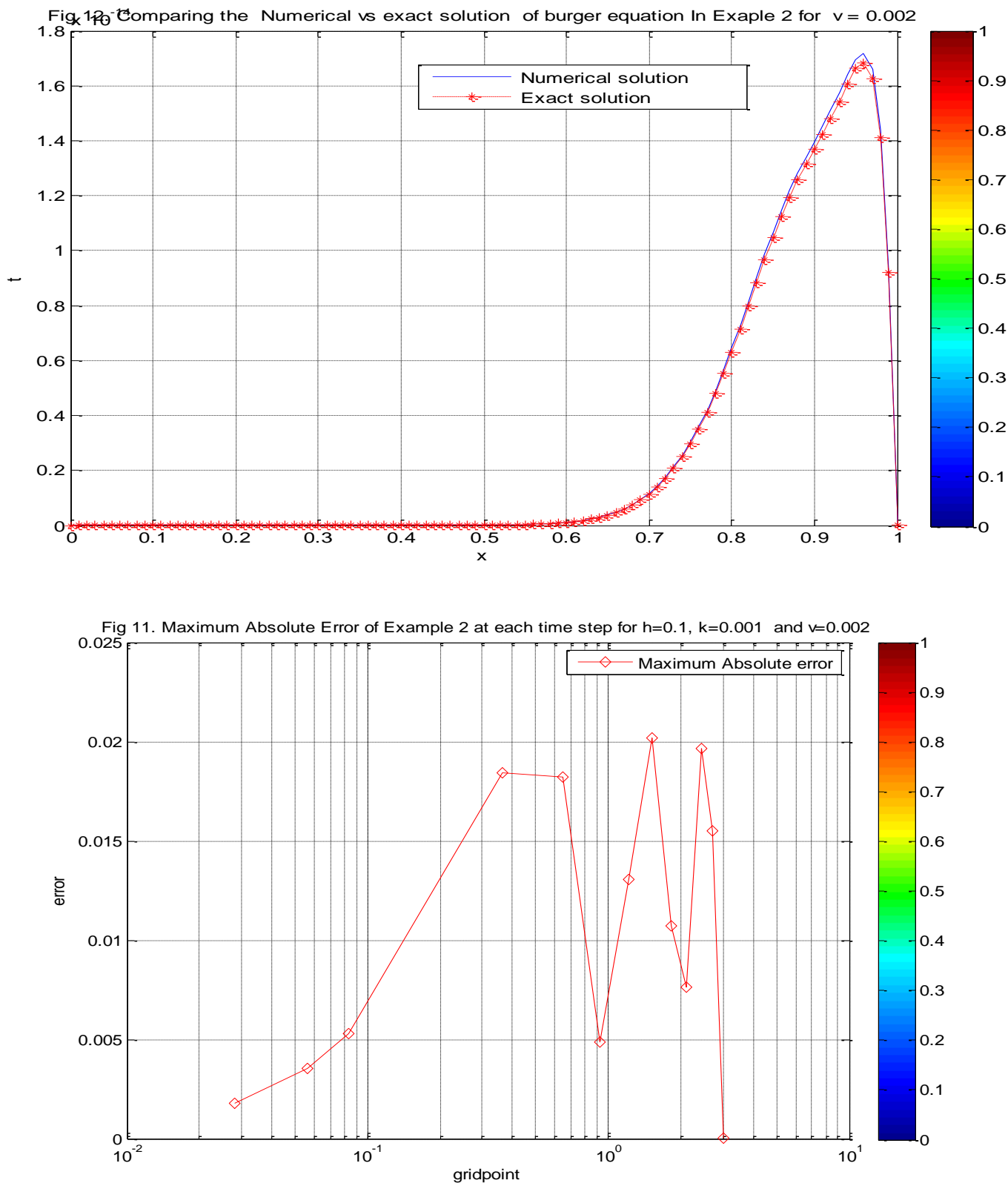

\section{DISCUSSION}

In this paper, weighted average based differential quadrature method is presented for solving one-dimensional homogeneous first-order non-linear parabolic partial differential equation (the Burger equation). To demonstrate the competence of the method, two model examples are solved by taking different values for step size h, and time step k. Numerical results obtained by the present method have been associated with numerical results obtained by the method in [1], [6], [31] and the results are summarized in Tables and graph. Moreover, in the present numerical computation, the result presented in Table 1 shows that Both root mean square error norm $\left(\mathrm{L}_{2}\right)$ and maximum absolute error norm $\left(L_{\infty}\right)$ are incases as the number of mesh point $M$ and $\mathrm{N}$ increases in both directions. In this case, the accuracy of the present method increases and it's superior to pre-existing. Again the result presented in Table 2 is also shows that Both root mean square error norm $\left(\mathrm{L}_{2}\right)$ and maximum absolute error norm $\left(\mathrm{L}_{\infty}\right)$ incases rapidly as the number of mesh points $\mathrm{M}$ decrease spatial direction and fixed grid number $\mathrm{N}$ temporal direction. In this case, the accuracy of the present method is rapidly increases. Hence the present method is superior to the methods that exist in literature review. Further, as shows in Figs. 1- 12, the

proposed method approximates the exact solution very well for different values of step length $h$ and time step $k$.To further verify the applicability of the planned method, graphs were plotted aimed for solution of Examples 1 and 2 as exact solutions versus the numerical solutions obtained by the present method showed by figs 3,7 , and 12 respectively indicate good agreement of the results, and proves the reliability of the present method. Also, Figs. 4, 8, and 11 are specifying the behavior of absolute error obtained by the present method within the effects of mesh sizes on the solution domain.

Published By: 
Hence, the numerical results presented in this paper validate the improvement of the proposed method over some of the existing methods described in the literature. Both the theoretical and numerical error bounds have been established. The results in the Tables are further confirmed that the are in agreement.

However the comparison among Table 2-Table 2 and the graphs of the numerical versus exact solution of one-dimensional Burger equation shows that the present method generates a more accurate result and it is superior to the method developed in [1],[6], [31] and It is approximate the exact solution very well.

\section{CONCLUSION}

A new approach, the weighted average based differential quadrature Method is using to solve the one-dimensional non-linear parabolic partial differential equation (Viscous Barger equation) numerically is presented in this study. Generally the comparison of the results obtained by the present method with other methods reveals that the present method is more convenient, reliable, and effective. Theoretical and mathematical error analysis is also developed in this study. As it can be seen that, the accuracy improves when the number of grid points is large. In a summary, the weighted average-based differential quadrature Method is a reliable method that is capable to solve the one-dimensional non-linear parabolic partial differential equation. Based on the findings, this method is well approximate and gives better accuracy of the numerical solution with a fixed time step, $k_{v}$ and large step size $h$.

\section{ACKNOWLEDGEMENTS}

The author wish to express his thank to the authors of literatures for the provision of initial idea for this work. Next to that, the author wish to express his thank to all his beloved friends; they kindness and helps be a great memory for me.

\section{REFERENCES}

1. Jie Zhao, Hong Li, Zhichao Fang, and Xue Bai. Numerical Solution of Burgers' Equation Based on Mixed Finite Volume Element Methods, Hindawi, Discrete Dynamics in Nature and Societ, Vol. 2020, 1-13,( 2020). [CrossRef]

2. N. Sugimoto. Burger's equation with a fractional derivative; hereditary effects on nonlinear acoustic waves. Journal of fluid mechanics, 225, 631-653, 1991. [CrossRef]

3. Solomon Bak, Philsu Kim, Xiangfan Piao, and Sunyoung Bu.Numerical solution of advection-diffusion type equation by modified error correction scheme. Journal of Advances in Difference Equations, (2018), 1-14. [CrossRef]

4. J. Bec, K. Khanin. Burger's turbulence. Physics reports, 447(1-2), 1-66, 2007. [CrossRef]

5. J. B. Bell, P. Colella, H. M. A .Glaz. second-order projection method for the incompressible Navier-Stokes equations. Journal of computational physics, 85, (1989)257-283. [CrossRef]

6. Amit Kumar Verma, Mukesh Kumar Rawani, Ravi P. Agarwal. On a seventh order convergent weakly $L$-stable Newton Cotes formula with application on Burger's equation. 2019

7. S. Gowrisankar, Srinivasan Natesan. An efficient robust numerical method for singularly Perturbed Burgers' equation Applied Mathematics and Computation 346 (2019). [CrossRef]

8. Sachin S. Wani and Sarita H. Thakar. Crank- Nicolson type method for burgers equation. International Journal of Applied Physics and Mathematics, 3(5) 2013 computational rate of convergence and theoretical estimates

9. J. M. Burgers. A mathematical model illustrating the theory of turbulence. Advances in Applied mechanics, 1, 171-199, 1948. [CrossRef]

10. S Mungkasi. Effects of a Logistic Reaction to Finite Difference Numerical Solutions of the Inviscid Burgers Equation, Indonesian Journal of Information Systems (IJIS), 2(2), 11-15, 2020[CrossRef]

11. Abdolrahman Yaghoobi, Hashem Saberi Najafi. A Fully Implicit Non-Standard Finite Difference Scheme for One Dimensional Burgers' Equation J. Appl. Res. Ind. Eng. 7(3) 301-312, 2020

12. A.H. Khater, R.S. Temsah, D.K. Callebaut. Numerical solutions for some coupled nonlinear evolution equations by using the spectral collocation method. Mathematical and Computer Modeling 48 (2008) 1237-1253[CrossRef]

13. J. B. Bell, Colella, P. Glaz, H. M. A second-order projection method for the incompressible Navier-Stokes equations. Journal of computational physics, 85, 257-283, 1989. [CrossRef]

14. E. Weinan , K.. Khanin, A. Mazel, Y.Sinai. Invariant measures for Burgers equation with stochastic forcing. Annals of Mathematics, 151(3), 877-96, 2000. [CrossRef]

15. G. W. Bluman, J. D. Cole .The general similarity solution of the heat equation Journal of mathematics and mechanics, 18, 1025-1042, 1969. [CrossRef]

16. G. B. Whitham. Linear, and nonlinear waves. John Wiley \& Sons, 2011.

17. H. Bateman, Some recent researches on the motion of fluids, Mon. Weather Rev. 43 (1915) 163-170. [CrossRef]

18. M.H. Protter, H.F. Weinberger, Maximum Principles in Differential Equations, Prentice Hall Inc., 1967.

19. A. H. Khater, R. S.Temsah, D. K Callebaut, . Numerical solutions for some coupled nonlinear evolution equations by using the spectral collocation method. Mathematical and Computer Modelling, 48(7-8), 2008, 1237-1253. [CrossRef]

20. J.G. Zheng, T.S. Lee, S.H. Winoto. A piecewise parabolic method for barotropic and Non-Barotropic two-fluid flows. International Journal of Numerical Methods for Heat \& Fluid Flow 18 ( 6) ,2008, 708-729[CrossRef]

21. M.K. Kadalbajoo, K.K. Sharma, A. Awasthi, A parameter-uniform implicit difference scheme for solving time-dependent Burgers' equation, Appl. Math. Comput. 170 (2005) 1365-1393[CrossRef]

22. Reza Abazaria, A. Borhanifar. Numerical study of the solution of the Burgers and coupled Burgers equations by a differential transformation method. Computers and Mathematics with Applications 59,2010, 2711-2722 [CrossRef]

23. R. Jiwari, R.C. Mittal, K. Kapil, . A .Sharma numerical scheme based on weighted average differential quadrature method for the numerical solution of Burgers' equation. Computation 219 (2013) 6680-6691[CrossRef]

24. A. Mignone, T. Plewa and G. Bodo. The Piecewise parabolic method for multidimensional Relativistic fluid dynamics, 2005[CrossRef]

25. Y. Zhang, , Z. Zong. Advanced differential quadrature methods. Chapman and Hall/CRC.The USA, 2009.

26. Cheong, H.T. (2015). Parallel Localized differential quadrature approach in boundary value Problem. Journal of math. Analysis and appl. 31(2):127-134

27. C. Shu, Differential Quadrature and its Application in Engineering, Athenaeum Press Ltd., Great Britain, 2000. [CrossRef]

28. D.Waston. Radial Basis Function Differential Quadrature Method for the numerical Solution of partial differential equations. The Aquila Digital Community, Dissertations, 2017.

29. Habtamu Garoma Debela. Exponential Fitted Operator Method for Singularly Perturbed Convection-Diffusion Type Problems with Nonlocal Boundary Condition. Hindawi, Abstract and Applied Analysis, Vol.2021, pp. 9. 2021. [CrossRef]

30. G. Fasshauer. Meshfree Application Method with MATLAB. Interdisciplinary Mathematical Sciences, 2007. [CrossRef]

31. S.S Xie, S. Heo, S. Kim, G. Woo, and S. Yi, Numerical solution of one-dimensional Burgers equation using reproducing kernel function, Journal of Computational and Applied Mathematics, 214(2), 2008, 417- 434[CrossRef]

32. K.W. Morton, D. F. Mayers. Numerical solution of partial differential equations: an introduction. Cambridge university press, 2005. [CrossRef]

33. B Neta. Numerical Solution of Partial Differential Equations. MA 3243 Lecture Notes 2003

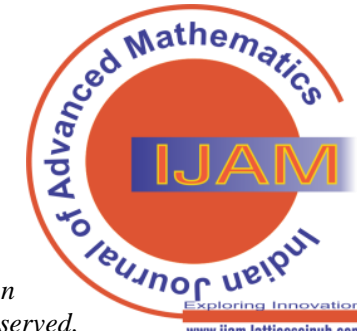




\section{AUTHORS PROFILE}

Kedir Aliyi Koroche, is Graduate B.sc in Mathematics from Ambo University in 2016 G.C. and M. Sc. in Mathematics(specialized in Numerical Analysis) from Jimma University in 2019 G.C. Now he works at Ambo University as Lecturer and Researcher. He has two published research paper. 